\title{
Éducation pour l'écodéveloppement au Brésil : promesses et incertitudes
}

Paulo Freire Vieira

\section{(2) OpenEdition \\ Journals}

Édition électronique

URL : http://journals.openedition.org/ere/4829

DOI : $10.4000 /$ ere.4829

ISSN : 2561-2271

Éditeur

Centr'ERE

Référence électronique

Paulo Freire Vieira, «Éducation pour l'écodéveloppement au Brésil : promesses et incertitudes », Éducation relative à l'environnement [En ligne], Volume 4 | 2003, mis en ligne le 14 septembre 2003 consulté le 16 juin 2020. URL : http://journals.openedition.org/ere/4829 ; DOI : https://doi.org/ 10.4000/ere.4829 


\title{
Éducation pour l'écodéveloppement au Brésil : promesses et incertitudes
}

\author{
Paulo Freire Vieira
}

Je tiens à remercier vivement mon épouse Anne-Sophie de Pontbriand-Vieira et mes amis Servane de Labrusse et Normand Brunet pour la contribution si généreuse qu'ils ont apportée à la mise en forme définitive de cet article.

La valeur d'un but ne tient pas à son accessibilité, mais à l'attraction magnétique de la direction qu'elle impose. C'est le voyage qui importe, plus que le point d'arrivée.

(Pierre Dansereau, 1999a)

1 Nous habitons une biosphère en évolution, régie par la loi d'entropie, ne fournissant qu'un milieu fragile et périssable à nos existences. Il est désormais évident qu'elle est en proie à des crises qui se succèdent rapidement. Ces tendances expriment le manque de conscience de nos responsabilités à l'égard de l'ampleur des destructions en cours. Comme si les problèmes socio-environnementaux d'aujourd'hui ne représentaient qu'une simple turbulence intempestive et temporaire, passible de traitement topique et segmenté, sans remettre en cause les sources de la maladie : la logique profonde qui sous-tend et commande la dynamique des sociétés contemporaines.

2 Les actions correctives se sont montrées jusqu'à présent ambiguës, fragmentées et peu capables de rendre compte de la complexité d'un effort coordonné de réduction des inégalités à l'intérieur de chaque pays et entre les pays, de contrôle démocratique des risques liés à l'évolution technique, ainsi que de création de rapports de symbiose authentique et durable avec la nature. En outre, les modes de raisonnement basés sur le paradigme analytique-réductionniste (Von Bertalanffy, 1968), encore dominant dans nos institutions d'enseignement et de recherche, sont loin de nous offrir de véritables instruments d'aide à la compréhension du monde et à la prise de décision politique. Ces modes font partie de la dynamique de dégradation généralisée de l'environnement biophysique et construit, ainsi que de son corollaire, l'aggravation dramatique du fossé entre riches et pauvres. 
3 Les initiatives qui se bornent à un aspect isolé de cette problématique complexe ne peuvent conduire à des solutions effectives, car il y a une unité fondamentale entre tous les grands systèmes de la nature et de la société. Dans la mesure où la résolution des problèmes environnementaux ne fait pas appel à des spécialisations mono- ou pluridisciplinaires, il nous faut un point de vue alternatif, englobant le processus coévolutif société-nature sous tous ses aspects. En d'autres termes, le phénomène qui nous occupe relève non de la juxtaposition de "facteurs » disciplinairement isolés, mais de leurs interdépendances au sein d'un système global constitué précisément par ces interactions elles-mêmes. Plutôt que de réduire de manière disjonctive les phénomènes complexes à tel ou tel aspect, l'approche systémique s'efforce au contraire de conjoindre ces aspects pour les intégrer et les contextualiser. Le défi est de réunifier les dimensions biologiques et socioculturelles à travers les interactions écosystémiques. Comme le rappelle si lucidement Pierre Dansereau (1994, p. 10), «il nous appartient donc de créer un nouveau modèle de solidarité biologique à l'échelle planétaire. Un tel projet repose sur les sciences de l'environnement, elles-mêmes unifiées par une pensée écologique ».

4 Cette nouvelle grille d'analyse transdisciplinaire nous amène à reposer :

les questions éternelles de notre origine, de la signification de la vie, des conséquences de notre action, de notre destinée. C'est dans ce contexte global que ces questions s'éclairent d'une lumière tout autre. Elle ne veut plus - et ne peut plus - séparer l'objet du sujet. Séparer la certitude des faits expérimentaux accumulés patiemment par la science, de la signification et de la finalité de l'action consciente et créatrice qui transforme le monde. (De Rosnay, 1975, p. 238)

5 Mais l'ouverture à une représentation systémique du monde et à la création d'une forme alternative d'organisation de la vie à l'échelle planétaire commence à peine à être incorporée dans nos conceptions et pratiques pédagogiques.

6 Dans cet article, nous apportons une modeste contribution à cette discussion en dressant, dans leurs grandes lignes, les contours d'une démarche d'éducation relative à l'environnement envisagée comme une composante à part entière de l'approche (systémique) d'écodéveloppement. Il s'agira de cerner la cohérence et l'originalité de ce concept ainsi que de mettre en relief les obstacles à sa mise en œuvre dans un contexte de mondialisation néo-libérale dont l'influence touche en profondeur toutes les dimensions des sociétés contemporaines. Parmi ces obstacles, nous soulignons la force d'inertie des démarches pédagogiques de transmission de connaissances fragmentées et décontextualisées au détriment de la mise en œuvre d'un nouveau mode de penser et d'agir, globaliste et synthétique, et d'une nouvelle sensibilité écoformatrice (Pineau, 2001a). En même temps, nous proposons quelques pistes préliminaires pour une évaluation critique d'ensemble des expériences d'éducation relative à l'environnement déclenchées au Brésil après le Sommet de la Terre tenu à Rio de Janeiro en 1992.

\section{Écodéveloppement}

7 Des controverses, apparemment interminables sur la façon jugée la plus féconde de définir les termes de référence pour la recherche d'alternatives de développement face à la crise planétaire de l'environnement, monopolisent encore les débats dans les universités, les agences gouvernementales, les réseaux associatifs et les médias. Chaque point de vue exprime des perceptions et attitudes diversifiées sur le rôle attribué à 
l'espèce humaine dans la biosphère, sur les sujets prioritaires de recherche scientifique et technologique ainsi que sur les lignes directrices les plus pertinentes de réforme socio-institutionnelle. En ce sens, nous devons faire face à un contexte idéologique marqué par le poids des controverses relatives aux systèmes de croyances et aux valeurs en jeu, et des incertitudes par rapport aux « consensus » déjà établis.

Les débats autour des concepts d'écodéveloppement et de développement durable font partie de cette nébuleuse environnementale. Le premier concept désigne ici une approche de planification et de gestion des interactions nature-société basée sur les sciences de l'environnement. Il s'agit d'une approche normative dans la mesure où une compréhension la plus rigoureuse possible de la dynamique co-évolutive (Norgaard, 1995) des systèmes sociaux et environnementaux est associée à la remise en cause éthique des styles de vie responsables de l'aggravation de la crise globale et à l'identification des espaces de manœuvre pour passer de la croissance indifférenciée et destructive à une croissance organique (Mesarovic et Pestel, 1974 ; Sachs, 1980, 1981 ; Glaeser, 1984, 1997 ; Bunge, 1989 ; Engel et Engel, 1990 ; Kothari, 1993 ; Goulet, 1995 ; Prades, 1995 ; Sauvé, 1996, 1997 ; Larrère et Larrère, 1997 ; Dansereau, 1999b).

De ce point de vue, les efforts de planification et de gestion doivent tenir compte de la nécessité de faire passer les certitudes de l'idéologie économique (Bourg, 1996) au crible de l'approche systémique des sciences de l'environnement. La grille d'analyse couvre des sujets apparemment aussi divers que les modèles actuels de consommation et les modes de vie ; les dynamiques d'appropriation, d'usage et de gestion économiquement viables des ressources naturelles et des territoires; les risques technologiques; la promotion de l'équité et de la coopération ainsi que la mise en œuvre de régimes de gouvernance. Remettant en cause l'autonomie supposée du cycle des activités économiques par rapport à l'état de l'environnement et aux autres dimensions de la vie sociale, l'approche d'écodéveloppement nous permet donc d'identifier, parmi les enjeux actuels de la crise environnementale, différents aspects d'une crise des logiques prédominantes dans l'organisation des sociétés contemporaines (Dag Hammarskjöld Foundation, 1975; Passet, 1979; Sachs, 1980, 1981 ; Morin et Kern, 2000). Cette approche marque ainsi un changement radical dans la conceptualisation des relations environnement-développement et se distingue de certaines versions du concept de développement durable inspirées par la théorie néo-classique des effets externes, soit une théorie considérée comme le fondement de l'économie de l'environnement.

Du point de vue de Godard (1998, p. 220-221) :

Analytiquement, l'écodéveloppement repose sur l'étude des interdépendances et des marges de liberté entre les principales variables de ce qui fut appelé des styles de développement. C'est sur cet échiquier du développement que se joue le jeu de l'harmonisation auquel doit se livrer le planificateur écodéveloppeur. Trois blocs de variables d'action sont distingués : le pôle de la demande sociale, autour du concept de style de vie, celui de l'offre (la gestion des ressources naturelles et l'approvisionnement en matières premières, l'approvisionnement énergétique, la localisation des activités et l'organisation de l'espace, les techniques, comprenant à la fois la technologie des produits et la technologie des procédés) et l'environnement proprement dit. Le jeu de l'harmonisation consiste à inventer une stratégie pour faire bouger de concert styles de vie et de consommation, styles technologiques, styles d'aménagement de l'espace, modes de gestion des ressources et approvisionnement énergétique dans un sens plus compatible avec la satisfaction des besoins fondamentaux des populations et la préservation de l'environnement. 
11 Selon cette conception, la destruction plus ou moins irréversible du patrimoine naturel et culturel est surtout liée au fonctionnement d'institutions de planification et de gestion héritières d'une cosmovision mécaniciste. Y sont inclus aussi bien les « impératifs » libéraux - soi-disant inexorables - de croissance matérielle illimitée et de maximisation de la rentabilité financière à court terme, que ceux de l'hypertrophie du contrôle étatique - autoritaire et technocratique - de l'économie, typiques de la tradition $\mathrm{du}$ "socialisme réel ». On constate, dans les deux cas, que les efforts de dynamisation du tissu socio-économique n'ont pas atteint l'objectif de pacification réelle et durable des relations que les êtres humains entretiennent entre eux et avec la nature. Selon Leonardo Boff (1996), les deux modèles ont :

rompu avec la terre, la réduisant à un réservoir de matières premières et ressources naturelles. Les êtres humains, réduits à des ressources humaines ou au capital humain, forment la grande armée de réserve, à la disposition des propriétaires des moyens de production (l'État ou le Capital). Ils ne sont plus en mesure d'entendre la Terre et la communauté cosmique à travers ses mille voix et paroles. (Boff, 1996, p. 109)

12 En fait, la dégradation progressive du tissu du vivant s'est intensifiée de façon dramatique ces derniers temps. Il ne fait guère de doute que la difficile transition du XXe au XXIe siècle et l'implosion inattendue du bloc socialiste ont entraîné le déplacement du pouvoir décisionnel de la sphère publique et politique vers les «milieux» financiers internationaux, privilégiant ainsi la poursuite obsessionnelle d'intérêts strictement privés (Passet, 1979 et 2000 ; Vivien, 1994).

13 Enfin, dans une perspective planétaire, nous considérons que la détérioration de l'environnement écologico-économique global constitue l'une des principales menaces à l'équilibre du système-monde. Cette détérioration est caractérisée par l'épuisement possible des ressources non renouvelables, l'amenuisement des ressources protéiques marines, la contamination des chaînes alimentaires par les produits chimiques agricoles et industriels et la possibilité d'une rupture de l'équilibre thermique du système énergétique terre/atmosphère.

14 Le fondement normatif de l'écodéveloppement suppose que les efforts voués à la satisfaction des besoins essentiels des populations (physiologiques, psychosociaux, économiques, éthico-politiques, spirituels) doivent s'appuyer sur le principe de subsidiarité et sur le fonctionnement d'une économie négociée et contractuelle - une économie du patrimoine naturel et culturel - sensible à la complexité des systèmes socioenvironnementaux (Morin et Kern, 2000) et à "l'inadéquation - toujours plus ample, profonde et grave - entre les savoirs séparés, fragmentés, compartimentés en disciplines, et les réalités ou problèmes toujours plus multidisciplinaires, transversaux, multidimensionnels, transnationaux, globaux, planétaires»(Morin, 2000a, p. 13). En outre, la défense du pluralisme technologique implique le développement de la capacité de faire un bilan et d'intégrer toute la richesse du savoir-faire des populations qui ont su maintenir, à travers le temps, une relation écologiquement prudente d'utilisation des écosystèmes et des paysages (Berkes, 1998 ; Gadgil, 1998, 1999 ; Singh et coll., 2000).

Dans ce contexte, les théoriciens de l'écodéveloppement considèrent la décentralisation et la «synthèse centralisée » comme des procédés complémentaires dans les prises de décision politique. La décentralisation renforce l'indépendance - la confiance en soi et l'autonomie des communautés - dans le cadre d'une co-gestion responsable des biens communs, ce qui suppose de subordonner l'économie aux finalités humaines et aux contraintes imposées par la recherche de résilience des écosystèmes 
(Ozbekhan, 1971; Dag Hammarskjöld Foundation, 1975; Godard et Sachs, 1975 ; Galtung, 1977 ; Colby, 1990 ; Berkes et Folke, 1998). La « synthèse centralisée » offre des atouts décisifs pour faire face aux incertitudes scientifiques, aux effets plus ou moins imprévisibles de la dynamique des systèmes socio-environnementaux et au pluralisme des visions du monde, des idéologies sociopolitiques et des options technologiques (Barel, 1984 ; Chaumont et Van Parijs, 1991 ; Sachs, 1995).

Exprimant l'émergence d'un nouveau principe de rationalité sociale, l'approche s'avère un outil capable d'assurer une articulation plus cohérente entre trois niveaux d'intervention. D'abord, celui de l'écologie intérieure menant à la rupture plus ou moins radicale avec notre façon rigide de percevoir le sens de la présence humaine dans l'univers. Ensuite, celui de l'écologie globale, avec une perception de plus en plus claire de la gravité des changements environnementaux globaux et du poids des asymétries Nord-Sud dans la constitution et le renforcement de ces tendances. Finalement, comme un effort d'articulation entre les deux premiers, le niveau intermédiaire des actions tendant à la création de systèmes de gestion intégrée de l'environnement (Glaeser, 1984 ; De Montgolfier et Natali, 1987; Berkes, 1989; Gadgil et Berkes, 1991; Bromley, 1992 ; Margerum, 1999; Vieira et Weber, 2002). Les stratégies d'écodéveloppement constituent ainsi des expérimentations locales, traduisant des objectifs globaux et exigeant, de ce fait, une conception innovatrice des processus éducatifs.

\section{Éducation pour l'écodéveloppement}

17 Les conceptions d'éducation relative à l'environnement véhiculées depuis les années 1970 reposent sur différents systèmes de valeurs, différentes épistémologies et conceptions du monde. Nous pouvons mentionner succinctement ici : une démarche préservationniste, de source néo-positiviste et valorisant la perspective des sciences de la nature; une démarche herméneutique, reposant sur une ontologie relativiste et une épistémologie constructiviste, où l'accent est mis sur le contenu symbolique des interactions sociales et sur la dimension d'une écologie intérieure et finalement, une démarche d'écologie politique, supposant une implication coordonnée des acteurs sociaux dans la création d'alternatives de développement local intégré. Dans celle-ci, l'approche systémique nous permet de déchiffrer la nature contradictoire et conflictuelle des configurations sociopolitiques constituant le cœur des problèmes étudiés. En ce sens, les connaissances sont construites et partagées au cours d'un processus de recherche coopérative, multiacteurs, préfigurant une modalité encore embryonnaire de recherche-action-formation communautaire (Sauvé, 1996, 1999, 2001; Anadón et coll., 2000 ; Liarakou et Flogaitis, 2000).

De notre point de vue, la conception d'éducation pour l'écodéveloppement intègre et dépasse les conceptions précédentes - dans le sens dialectique de dépasser tout en conservant certaines caractéristiques jugées nécessaires, mais insuffisantes. Nourrie par les avancées de la recherche en sciences cognitives, cette conception prend ses racines dans la systémique (Buckley, 1967; Von Bertalanffy, 1968; Varela, 1989; Andreewsky, 1991 ; Varela et coll., 1993 ; Le Moigne, 1995 ; Ragou et Souchon, 2000 ; Jollivet et Pavé, 2002).

19 Nous partageons avec la conception d'éducation relative à l'environnement développée par Lucie Sauvé et ses collaborateurs de l'Université du Québec à Montréal le présupposé 
selon lequel il s'agit d'un nouveau paradigme éducationnel, s'inscrivant dans une pédagogie de projets :

l'éducation relative à l'environnement correspond à un projet éducatif spécifique, mais ample, dont le large spectre d'objectifs couvre les divers aspects du rapport à l'environnement. Parmi ces derniers, mentionnons la quête fondamentale d'un sens à notre être dans le monde, la compréhension de notre ancrage dans la nature, la définition de notre niche écologique humaine dans l'écosystème global, la critique sociale des rapports de pouvoir liés à l'environnement et la mise en projets collectifs d'écodéveloppement. (Sauvé, 2001, p. 33)

L'enjeu décisif est de rendre des individus, groupes sociaux et communautés toujours plus en mesure d'appréhender, de comprendre et de faire face - de façon lucide, théoriquement informée, éthiquement réfléchie et politiquement responsable - aux sources structurelles de la crise contemporaine de l'environnement et des styles de développement. Diffuser l'histoire écologique de l'humanité (Morin et Piatelli-Palmarini, 1974 ; Dansereau, 1999b, 1999c; Morin et Kern, 2000), approfondir la critique de l' idéologie économique (Bourg, 1996) et de son influence sur les modes de vie contemporains et promouvoir la révérence pour la vie (Schweitzer, 1976) et l' écocitoyenneté planétaire (De Rosnay, 1975 ; Dansereau, 1999d; Morin, 2000a, 2000b) par des actions concrètes sur le terrain constituent les dimensions essentielles de ce nouveau concept pédagogique.

21 Les expérimentations pédagogiques menées à l'échelle communautaire correspondent à une recherche de solutions réfléchies et négociées entre personnes ne partageant pas obligatoirement les mêmes croyances, valeurs, codes de conduite, et donc projets de vie, mais qui seraient capables - en principe - d'arriver à reconnaître, à travers l'idée d'un patrimoine à conserver et à transmettre aux générations futures, une logique d'action collective radicalement différente de la longue tradition d'ingérence technocratique qui a marqué - de façon brutale - le déroulement du XXe siècle, le "siècle de l'impérialisme " (Sader, 2000). La gestion de nos rapports multidimensionnels avec le patrimoine naturel et culturel devient ainsi un espace permanent d'apprentissage de l'art d'étendre la conscience et de recréer la vie quotidienne, par la prise en compte d'une responsabilité commune à long terme.

Deux acceptions complémentaires du terme patrimoine sont proposées par Jean de Montgolfier et Jean-Marc Natali (1987) : il peut être conçu comme un bien susceptible de conserver dans le futur des potentialités d'adaptation à des usages non prévisibles dans le présent (visée économique) et aussi comme un ensemble d'éléments matériels et immatériels qui concourent à sauvegarder l'autonomie et l'identité de leur titulaire ainsi que son adaptation au fil du temps dans un univers instable (visée sociopolitique).

23 Assurant une certaine continuité entre le passé et le futur, cette démarche de gestion se fonde largement sur l'incertitude et les controverses scientifiques, et résulte d'un double éclairage, à la fois objectif et subjectif: la qualité des milieux en tant que patrimoine est considérée d'une part, comme un objet physique (point de vue scientifique et technique) et d'autre part, comme la résultante d'un engagement (positif ou négatif ) d'un ensemble d'acteurs sociaux agissant au sein de l'unité écologicohumaine concernée (point de vue sociopolitique et relationnel). Une grille d'analyse patrimoniale permet d'établir des diagnostics participatifs des écosystèmes et des paysages et aussi d'élaborer des stratégies plurielles d'écodéveloppement à long terme, adaptées aux particularités de chaque contexte socioécologique. Pris au sens large, l'environnement représenté comme patrimoine devient alors facteur d'amplification 
d'identités personnelles et collectives ainsi qu'un élément structurant d'une nouvelle culture d'apprentissage permanent (Friedmann et Abonyi, 1976 ; Ribeiro, 1981 ; Berkes, 1989 ; Bromley, 1992 ; Godard, 1997 ; Berkes et Folke, 1998 ; Gadgil, 1998, 1999 ; Diduck, 1999 ; Vieira et Weber, 2002).

Cette quête obstinée de lucidité dans la recherche d'un nouveau cadre de référence éthique correspond au besoin de :

situer tout événement, information ou connaissance en relation d'interdépendance avec son environnement - culturel, social, économique, politique, et bien sûr naturel. Il nous amène non seulement à situer un événement dans son contexte, mais nous incite, par ailleurs, à percevoir comment ce contexte le modifie et l'explique d'une autre façon. (Morin, 2000a, p. 25)

En outre, la capacité d'apprentissage du titulaire (individus, collectivités, communautés) et sa capacité d'innovation par le biais d'un processus incertain d'essaiserreurs jouent un rôle essentiel dans le maintien et l'évolution de son patrimoine. Celui-ci devrait être géré dans le respect des cycles et des rythmes auxquels il est soumis, des échelles spatio-temporelles des phénomènes biologiques mis en jeu, des seuils qui ne peuvent être dépassés sans entraîner un risque de dégradation plus ou moins irréversible à moyen ou à long terme.

\section{Les acquis de l'expérience brésilienne}

Une décennie après le Sommet de Rio, tandis que se poursuit la période de maturation de la prise de conscience écologique déclenchée au cours des années 1980, s'intensifie la transmission, par les médias et les réseaux d'organisations non gouvernementales écologistes, des données concernant les dommages infligés au milieu biophysique. Dans la plupart des cas, et peut-être à cause du caractère flou du concept d'environnement, nous pouvons identifier dans cette mouvance :

la force du naturalisme naïf et émotionnel dans les représentations communes et l'écart considérable qui existe entre l'écologisme quotidiennement vécu, et les points de vue hautement rationalisés des discours tenus tant par les spécialistes de l'écologie scientifique que par les leaders de l'écologie politique. (Lascoumes, 1994, p. 39).

Malgré la surenchère des colloques voués à l'élargissement progressif d'une réflexion commune, à la quête d'une meilleure concertation des acteurs sociaux impliqués et aussi à la recherche d'une meilleure représentation face aux pouvoirs publics, le changement de mentalité et de style de vie des citoyens s'accélère à peine sous l'angoisse croissante face à la montée des nuisances en milieu urbain et à quelques accidents écologiques majeurs. Cependant, si quelques indices de contestation peuvent être repérés parmi certaines fractions des classes moyennes «instruites", ils expriment, en règle générale, une sorte de contestation rhétorique et ambivalente dans le système plutôt qu'une véritable contestation $d u$ système. Leurs propres conditionnements immédiats, quotidiens et privés, soigneusement ajustés aux contraintes posées par le (dés)ordre établi (« le confort et l'indifférence », dirait Pierre Dansereau), font écran à leur perception de l'urgence de la question environnementale.

Cependant, il nous faut reconnaître que des efforts considérables ont été entrepris par le ministère de l'Éducation nationale au Brésil pour intégrer la problématique de l'environnement dans le système d'enseignement public du premier et du second cycles. Ajoutons à cela les nouvelles perspectives d'insertion créative de l'éducation 
pour l'écodéveloppement dans le processus de mise en œuvre de l'Agenda 21 brésilien et la diversification des lignes de financement pour des actions pédagogiques au niveau communautaire issues du Fonds national de l'Environnement (FNMA), relié au Ministère de l'Environnement.

Toutefois, la société brésilienne ressent encore aujourd'hui l'absence de volonté politique pour donner vie à une stratégie cohérente d'éducation pour l'écodéveloppement sensible à l'étonnante diversité biologique et culturelle du pays. Les expériences éducatives relatives à l'environnement restent marquées par l'incapacité chronique de transformer les discours génériques sur le saccage de la nature et les exhortations moralisantes pour la sauvegarde de la planète, en stratégies efficaces de changement durable des perceptions et pratiques quotidiennes des citoyens. En plus, si les rôles attribués aux éducateurs - et les critères de formation des enseignants - sont encore mal définis, cette situation tend à s'aggraver du fait de la grande diversité actuelle des théories sur le processus d'apprentissage et des approches pédagogiques (Carr et Kemmis, 1986 ; Robottom et Hart, 1993 ; Diduck et Sinclair, 1997 ; Diduck, 1999 ; Sauvé, 1999 ; Liarakou et Flogaitis, 2000).

En même temps, sous la prolifération vertigineuse de programmes d'enseignement universitaire en sciences de l'environnement, se cache un développement insuffisant des bases théoriques et méthodologiques de la recherche sociale en environnement. Ceci reflète une certaine difficulté à associer l'être humain en tant que facteur écologique induisant la persistance d'un préjugé «biologisant et préservationniste " (Reigota, 1995). Dans ce cas, la pédagogie de l'environnement est très souvent confondue avec un effort de transmission « livresque » de connaissances mono- et, à la limite, pluri- disciplinaires et de conception d'ateliers thématiques fragmentés, sans atteindre le seuil des changements comportementaux qui décèlent une perception systémique du monde et de la vie.

Il faut aussi mentionner les cloisonnements, à des degrés variables, entre les institutions, rendant extrêmement difficiles l'établissement et la consolidation institutionnelle d'équipes d'enseignement et de recherche inter- et transdisciplinaires. En fonction d'une dispersion considérable des groupes de chercheurs, aussi bien que de l'hégémonie de la tradition de l'individualisme académique, très peu de laboratoires d'éducation relative à l'environnement disposent aujourd'hui d'une masse critique suffisante pour jouer le rôle de centres de rayonnement, capables de faire progresser les bases théoriques et de stimuler les applications de ce nouveau paradigme éducatif en se basant sur une méthodologie rigoureuse. Les carences du système public de financement de la recherche scientifique et la réticence des chercheurs spécialisés et des administrations des facultés en ce qui concerne les défis épistémologiques et éthiques suscités par la complexité systémique et par la «fin des certitudes" apparaissent évidentes (Prigogine, 1996 ; Dansereau, 1999d ; Laszlo, 2001).

Heureusement, quelques hirondelles dans le ciel d'hiver nous renvoient sur des pistes plus porteuses d'innovations, ouvrant ainsi de multiples chantiers de recherche-actionformation compatibles avec la démarche présentée dans cet article. Sans aucune prétention à l'exhaustivité, nous mettons en évidence :

- le projet de coopération internationale Educación ambiental en Amazonia (EDAMAZ), conçu pour le développement de programmes de formation en éducation relative à l'environnement (Anadón et coll., 2000) ; 
- la mise en œuvre d'une démarche rigoureuse de Participation-action pour la construction de la connaissance (PROPACC), vouée à la formation d'enseignants et d'agents du secteur public (Medina et Santos, 2000);

- les expériences d'action pédagogique au niveau communautaire menées par les chercheurs du Laboratoire d'éducation environnementale en zones côtières de l'Université de la Vallée du Itajaí (CTTMAR-UNIVALI) (Matarezi et coll., 2003) ;

- la recherche de nouveaux outils pédagogiques coordonnée par Genebaldo Freire Dias (1998) au Centre de Recherches de l'Université Catholique de Brasília ;

- les expérimentations avec l'approche d'évaluation participative au niveau local d'écosystèmes qui sont partie prenante d'une stratégie de création en réseau d'Agendas 21 locaux dans les zones côtières (IBAMA, 1998 ; Vieira, 2003) ;

- la consolidation institutionnelle du Programme de formation (DEA) en développement et environnement (PRODEMA), reliant une dizaine d'universités dans la région nord-est du pays ;

- l'évaluation d'ensemble des outils de pédagogie de l'environnement utilisés dans le pays par l'Institut ECOAR pour la Citoyenneté (Trajber et Costa, 2001);

- l'axe d'éducation pour la paix diffusé au sein de l'Université Holistique Internationale de Brasília / UNIPAZ par Pierre Weil (2001).

\section{Pour une culture de la non-violence active}

Le potentiel, les risques et les limites d'une pédagogie de l'écodéveloppement deviennent plus visibles si nous prenons comme point de référence la distinction faite par Johan Galtung $(1978,1985,1996)$, dès les années 1970, entre violence personnelle ou directe et violence structurelle, de caractère plus diffus et abstrait. Cette dernière constitue un sous-produit des formes spécifiques - admises comme légitimes même sous la toile de fond des démocraties libérales modernes - d'organisation de la vie collective qui contredisent, de façon constitutive et paradoxale, l'universalité et l'indivisibilité des principes fondamentaux dégagés par la Charte universelle des droits de l'Homme.

Une option contre-hégémonique, encore en cours de légitimation, associe l'intelligence de la complexité (Morin et Le Moigne, 2000) à une conscience toujours plus claire des limites écologiques et sociales de la civilisation contemporaine, ainsi qu’à l'institutionnalisation de formes d'action collective dérivées des concepts de nonviolence active et d'aide mutuelle (Kropotkin, 1908; Gandhi, 1971; Woodcock, 1975; Nisbet, 1982 ; Fischer, 1992). Toutefois, il nous faut reconnaître que les efforts de mise en œuvre de ces principes sont, jusqu'à présent, restés fragmentés, dans le cadre d'une contre-culture embryonnaire (Meadows et Meadows, 1972; Engel et Engel, 1990; UNESCO, 1996 ; Morin, 2000a ; Singh et coll., 2000).

De notre point de vue, les principaux risques de dérive dans les applications de cette nouvelle conception de la mission éducative sont reliés aux croyances à des principes et des solutions qui seraient tenus pour évidents ou "naturels", définitivement inscrits dans la réalité sociale comme étant les "meilleurs ", et pourraient être imposés à toute personne ou collectivité en tant qu'expression d'un nouveau code universel de moralité (encore un!) ou d'une nouvelle vérité civilisatrice. Une valorisation attentive des résultats de la recherche sociale empirique sur le pluralisme inné des codes de conduite et des styles de vie des différentes cultures humaines nous amène, tout d'abord, à distinguer les positions respectives du chercheur et du moraliste ; par la suite, à mettre en relief la proéminence de la sphère politique, siège du débat démocratique et non 
dogmatique sur les risques socialement acceptables des stratégies de développement et sur les scénarios pour le futur considérés désirables et possibles du point de vue socioenvironnemental.

En s'inspirant d'Edgar Morin, de Pierre Dansereau et de David Bohm, cette nouvelle conception pédagogique nous semble constituer, avant tout, un effort coordonné de réforme de la pensée (et par conséquent une remise en cause éthique): d'une part, l'éducation pour l'écodéveloppement cherche à affiner notre perception des limites d'une connaissance fragmentée du monde, incapable de réunir les parties au tout et viceversa, en rendant ainsi compte de la complexité des processus de co-évolution hommenature ; d'autre part, elle cherche à élucider comment nous pourrions concilier la prise de conscience de l'altérité, le respect de la diversité des façons de donner un sens à l'existence et la formation de citoyens toujours mieux préparés à habiter de façon compatissante la Terre-Patrie (Bohm 1988; Bohm et Edwards, 1991; Vieira, 1998; Dansereau, 1999b, 1999d; Monod, 2000 ; Morin, 2000a, 2000b ; Morin et Kern, 2000 ; Weber, 2002).

La condition humaine est assumée ici dans sa totalité dynamique et multidimensionnelle, dépassant certaines dichotomies traditionnelles de la pensée analytique cartésienne : l'implication et la distanciation, l'affectivité et la rationalité, la recherche et l'action, l'autoformation et l'hétéroformation, la science et l'art, la connaissance scientifique et la connaissance écologique locale (Ardoino, 1980 ; Pineau, 1992, 2001a, 2001b; Barbier, 1996).

\section{Au cœur du débat : les préalables pour viabiliser cette démarche}

38 Malgré la prolifération de projets mis en œuvre dans différentes biorégions de la planète, l'impression qui prédomine est que cette démarche pédagogique heurte de front le « cours du monde». Elle semble défier les tendances lourdes qui président à la dynamique actuelle de globalisation des circuits économiques et financiers, d'homogénéisation culturelle et de détérioration progressive des relations Nord-Sud. N'est-il pas aujourd'hui évident que la violence structurelle impose la dissémination massive, à l'échelle planétaire, d'un style de développement dont la valeur suprême réside dans la marchandisation progressive de tous les domaines de l'existence humaine? Cette crise de "sens" des actions collectives, cette inversion tragique entre l'instrument et les valeurs est largement aggravée par les carences en informations techniques et en intégration des connaissances scientifiques, par la crise récurrente de crédibilité sociale de l'establishment universitaire, ainsi que par le fait - le dernier et non le moindre - qu'une vision alternative du développement des sociétés contemporaines attire pour le moment l'intérêt d'un segment nettement minoritaire des habitants de la planète.

39 Au niveau des États-nations, l'absence de volonté politique réelle d'avancer dans cette direction constitue aussi un blocage décisif. D'une part, l'écodéveloppement implique l'existence d'un espace d'autonomie locale des populations concernant les options de développement; d'autre part, il présuppose la conception de nouveaux systèmes participatifs de planification et de gestion. Cet effort de recherche tourné vers la création, à l'échelle planétaire, de cultures de la paix - avec soi-même, avec les autres et 
avec la nature (Weil, 1987, 2001 ; UNESCO, 1996) - ne peut devenir réalité qu'au moyen d'un certain interventionnisme de la part des États-nations. Par conséquent, il s'avère inconcevable en l'absence de réseaux coopératifs reliant la société civile, l'état et le marché. Alors, que faire ? Où trouver les points de bifurcation du système, les espaces de manœuvre, s'il en reste, pour l'enracinement d'un nouveau mode de régulation ? Ce sont là des questions difficiles.

Il nous faut reconnaître la dramatique disproportion des forces sociales par rapport à ces tendances lourdes, à un moment historique où, apparemment, les clés de la culture n'ont plus le pouvoir d'ouvrir de nouvelles portes, de faire émerger de nouveaux horizons et d'inspirer des groupes porteurs d'avenir. Dominique Bourg (1996, p. 118) reconnaît à son tour :

qu'il est difficile de faire preuve d'un optimisme béat. Une évolution lente des esprits accompagnée de réformes graduelles parviendra peut-être à infléchir suffisamment le cours des choses; mais on peut en douter. Il est malheureusement beaucoup plus probable qu'il faille compter sur la vertu pédagogique de quelques catastrophes pour ébranler nos mentalités et nos structures sociales.

41 Néanmoins, des espaces de résistance non violente à la barbarie du présent pourraient devenir plus visibles par le biais de la recherche transdisciplinaire. Nous devrions reconnaître aussi qu'un diagnostic lucide des impasses actuellement rencontrées dans la formation des éducateurs et des citoyens sensibles à une approche systémique et globale des réalités ne peut continuer à se faire dans un contexte d'activisme fragmenté, contraire aux (dures) exigences d'élaboration théorique et méthodologique, aux défis présentés par les incertitudes scientifiques et à la nécessité de préserver la diversité culturelle, le pluralisme et la tolérance comme des acquis fondamentaux du processus civilisateur (Ribeiro, 1981). Le passage par un certain recul tactique devient ainsi - peut-être - inéluctable.

Il nous faut ainsi non seulement repenser les fondements et la légitimité des pratiques d'écoformation en cours, mais encore enrayer la tendance à l'émiettement, à la dispersion et à la discontinuité des projets. Il est temps maintenant que ces pratiques atteignent un niveau supérieur de cohérence. La constitution de réseaux d'acteurs dans lesquels les problèmes d'identité individuelle, de développement personnel et d'action collective se superposent s'avère une possibilité intéressante, dans la mesure où la plupart des participants aux contre-cultures en formation semblent mener leurs vies sans essayer de convertir quiconque ou d'attirer l'attention sur eux (Laszlo, 2001).

Mettre en fonctionnement ce tissu interactif innovateur exigera des individus, groupes et communautés de base un effort redoublé d'expansion de la conscience, de dépassement d'une vision trop intellectualiste de l'agir humain (Bohm et Edwards, 1991). Ce constat peut devenir le point de départ d'une remise en cause plus rigoureuse des pratiques instituées au Brésil au nom de l'éducation relative à l'environnement. Il pourra éventuellement jalonner la voie conduisant à des expériences de transmutation graduelle, mais effective, de notre connaissance rationnelle et fragmentée du monde en sagesse, en discernement, en célébration de la vie, en art de vivre en paix (Weil, 1987, 2001 ; Bohm, 1988; Sjöberg, 1988; Bohm et Edwards, 1991; Varela, 1993, 1996; Maturana, 1996 ; Magro et coll., 1997 ; Vieira, 1998).

Malgré le visage sombre de notre temps, l'avenir reste indéterminé. Les êtres humains n'ont pas le pouvoir de modeler l'histoire à leur guise, mais ils ont celui de faire ce qui est possible. "Soyez vous-même le changement que vous souhaitez voir dans le 
monde", nous recommandait le Mahatma Gandhi. En conservant la volonté d'agir collectivement même devant la formidable coalition des intérêts établis, la force d'inertie des modes de raisonnement linéaires et la toute-puissance atteinte par le capitalisme planétaire, c'est maintenant un travail plus tenace sur soi-même qu'il est devenu urgent d'entreprendre.

\section{BIBLIOGRAPHIE}

Anadón, M., Sauvé, L., Carrasco, M.T. et Boutet, A. (2000). Quand évaluer c'est apprendre ensemble : une expérience d'évaluation dans l'action. Éducation relative à l'environnement : Regards - Recherches - Réflexions, 2, 31-47.

Andreewsky, E. (1991). Systémique et cognition. Paris : Dunod.

Ardoino, J. (1980). Éducation et relations. Introduction à une analyse plurielle des situations éducatives. Paris : Gauthier-Villars.

Barbier, R. (1996). La recherche-action. Paris : Anthropos. Barel, Y. (1984). La société du vide. Paris : Seuil.

Berkes, F. (1998). Sacred ecology. Traditional ecological knowledge and resource management. Philadelphia : Taylor \& Francis.

Berkes, F. (dir.). (1989). Common property resources. Ecology and community-based sustainable development. London : Belhaven Press.

Berkes, F. et Folke, C. (dir.). (1998). Linking social and ecological systems. Management practices and social mechanisms for building resilience. Cambridge : Cambridge University Press.

Boff, L. (1996). Dignitas Terrae. Ecologia : grito da terra, grito dos pobres. São Paulo : Ática.

Bohm, D. (1988). La danse de l'esprit. La Varenne : Les Éditions Séveyrat.

Bohm, D. et Edwards, M. (1991). Pour une révolution de la conscience. Paris : Éditions du Rocher.

Bourg, D. (1996). Les scénarios de l'écologie. Paris : Hachette.

Bromley, D.W. (dir.). (1992). Making the commons work. Theory, practice and policy. San Francisco : ICSP.

Buckley, W. (1967). A sociologia e a moderna teoria dos sistemas. São Paulo : Cultrix.

Bunge, M. (1989). Development and the environment. In E.F. Byrne et J.C. Pitt (dir.), Technological transformation: contextual and conceptual implications. The Hague : Kluwer, p. 285-304.

Carr, W. et Kemmis, S. (1986). Becoming critical. Education knowledge and action research. London : Taylor \& Francis.

Chaumont, J.M. et Van Parijs, P. (1991). Les limites de l'inéluctable. Penser la liberté au seuil du troisième millénaire. Bruxelles : De Boeck.

Colby, M.E. (1990). The evolution of paradigms of environmental management in development. Washington : The World Bank. 
Dag Hammarskjöld Foundation. (1975). What now ? Uppsala : DHF.

Dansereau, P. (1999a). A ética ecológica e a educação para o desenvolvimento sustentável. In P.F. Vieira et M.A. Ribeiro, (dir.), Ecologia humana, ética e educação. A mensagem de Pierre Dansereau. Porto Alegre et Florianópolis : Pallotti et APED, p. 23-62.

Dansereau, P. (1999b). A ecologia e a escalada do impacto humano. In P.F. Vieira et M.A. Ribeiro (dir.), Ecologia humana, ética e educação. A mensagem de Pierre Dansereau. Porto Alegre et Florianópolis : Pallotti et APED, p. 189-218.

Dansereau, P. (1999c). A terra dos homens e a paisagem interior. Belém : UFPA-NAEA. Dansereau, P. (1999d). Uma preparação ética para a mudança global. In P.F. Vieira et M.A. Ribeiro (dir.), Ecologia humana, ética e educação. A mensagem de Pierre Dansereau. Porto Alegre et Florianópolis : Pallotti et APED, p. 299-372.

Dansereau, P. (1994). L'envers et l'endroit. Le besoin, le désir et la capacité. Montréal : Musée de la civilisation.

De Montgolfier, J. et Natali, J.-M. (1987). Le patrimoine du futur. Approches pour une gestion patrimoniale des ressources naturelles. Paris : Économica.

De Rosnay, J. (1975). Le macroscope. Vers une vision globale. Paris : Seuil.

Dias, G.F. (1998). Educação ambiental. Princípios e práticas. São Paulo : Gaia.

Diduck, A. (1999). Critical education in resource and environmental management : learning and empowerment for a sustainable future. Journal of Environmental Management, 57, 85-97.

Diduck, A. et Sinclair, J. (1997). The concept of critical environmental assessment education. The Canadian Geographer, 41(3), 294-307.

Engel, J.R. et Engel, J.G. (dir.). (1990). Ethics of environment and development. Global challenge and international response. Tucson : The University of Arizona Press.

Fischer, L. (1992). Gandhi. Sua vida e mensagem para o mundo. São Paulo : Martin Claret.

Friedmann, J. et Abonyi, G. (1976). Social learning : a model for policy research. Environment and Planning, 8, 927-940.

Gadgil, M. (1999). New meanings for old knowledge : the People's Biodiversity Registers Programme. Bangalore : CES.

Gadgil, M. (1998). Conservation : Where are the people ? The Hindu Survey of the Environment '98, 107-137.

Gadgil, M. et Berkes, F. (1991). Traditional resource management systems. Resource Management and Optimization, 8(3-4), 127-141.

Galtung, J. (1996). Cultural peace : some characteristics. In UNESCO, (dir.), From a culture of violence to a culture of peace. Paris : UNESCO, p. 75-92.

Galtung, J. (1985). Sobre la paz. Barcelona : Fontamara.

Galtung, J. (1978). On violence in general and terrorism in particular. Oslo : University of Oslo. Galtung, J. (1977). Self-Reliance. A strategy for development. London : Bogle L'Ouverture.

Gandhi, M.K. (1971). Minha vida e minhas experiências com a verdade. Rio de Janeiro : Edições O Cruzeiro.

Glaeser, B. (1997). Environnement et agriculture. L'écologie humaine pour un développement durable. Paris : L'Harmattan. 
Glaeser, B. (dir.). (1984). Ecodevelopment. Concepts, projects, strategies. Oxford : Pergamon Press. Godard, O. (1998). L'écodéveloppement revisité. Économies et Sociétés, 36(1), 213-229.

Godard, O. (1997). A gestão integrada dos recursos naturais e do meio ambiente : conceitos, instituições e desafios de legitimação. In P.F. Vieira et J. Weber (dir.), Gestão de recursos naturais renováveis e desenvolvimento. Novos desafios para a pesquisa ambiental. São Paulo : Cortez, p. 201-266.

Godard, O. et Sachs, I. (1975). L'environnement et la planification. In J. Barrau (dir.), Environnement et qualité de la vie. Paris : Guy le Prat, p. 207-247.

Goulet, D. (1995) Development ethics. A guide to theory and practice. Nova Iorque : The Apex Press. IBAMA. (1998). Diagnóstico ambiental participativo do Vale do Ribeiro e Litoral Sul de São Paulo. São Paulo : Secretaria de Estado do Meio Ambiente.

Jollivet, M. et Pavé, A. (2002). O meio ambiente : questões e perspectivas para a pesquisa. In P.F. Vieira et J. Weber (dir.), Gestão de recursos naturais renováveis e desenvolvimento. Novos desafios para a pesquisa ambiental. São Paulo : Cortez, p. 53-112.

Kothari, R. (1993). Growing amnesia. An essay on poverty and the human consciousness. New Delhi : Viking.

Kropotkin, P. (1908). Mutual aid : a factor of evolution. London : William Heineman.

Larrère, C. et Larrère, R. (1997). Du bon usage de la nature. Pour une philosophie de l'environnement. Paris : Aubier.

Lascoumes, P. (1994). L'éco-pouvoir. Environnements et politiques. Paris : La Découverte.

Laszlo, E. (2001). Macrotransição. O desafio para o terceiro milênio. São Paulo : Axis Mundi.

Le Moigne, J.L. (1995). Les épistémologies constructivistes. Paris : PUF.

Liarakou, G. et Flogaitis, E. (2000) Quelle évaluation pour quelle éducation relative à

l'environnement ? Éducation relative à l'environnement : Regards - Recherches - Réflexions, 2, 13-30.

Magro, C., Graciano, M. et Vaz, N. (1997). Humberto Maturana. A ontologia da realidade. Belo Horizonte : Editora da UFMG.

Margerum, R.D. (1999). Integrated environmental management : the foundation for successful practice. Environmental Management, 24, 151-166.

Matarezi, J., Bonilha, L.E.C. et Mentges, T. (2003). A educação ambiental comunitária no litoral brasileiro e o papel da universidade. In P.F. Vieira (dir.), Conservação da diversidade biológica e cultural em zonas costeiras. Enfoques e experiências na América Latina e no Caribe. Florianópolis : APED Editora, p. 185-234.

Maturana, H. (1996). El sentido de lo humano. Santiago : Dolmen/Mundo Abierto.

Meadows, D. et Meadows, D. (1972). The limits to growth. New York : Universe Books.

Medina, N.M. et Santos, E.C. (2000). Educação ambiental. Uma metodologia participativa de formação. Petrópolis : Vozes.

Mesarovic, M.D. et Pestel, E. (1974). Stratégie pour demain. Paris : Seuil.

Monod, T. (2000). Et si l'aventure humaine devait échouer. Paris : Bernard Grasset. Morin, E. (2000a). A cabeça bem feita. Rio de Janeiro : Bertrand Brasil.

Morin, E. (2000b). Os sete saberes necessários à educação do futuro. São Paulo : Cortez.

Morin, E. et Kern, A.B. (2000). Terra-Pátria. Porto Alegre : Sulina. 
Morin, E. et Le Moigne, J.L. (2000). A inteligência da complexidade. São Paulo : Fundação Peirópolis.

Morin, E. et Piatelli-Palmarini, M. (dir.). (1974). L'unité de l'homme. Pour une anthropologie fondamentale. Paris : Seuil.

Nisbet, R. (1982). Os filósofos sociais. Brasília : Editora da UnB.

Norgaard, R.B. (1995). Development betrayed. The end of progress and a coevolutionary revisioning of the future. London : Routledge.

Ozbekhan, H. (1971). Planning and human action. In P. Weiss (dir.), Hierarchically organized systems in theory and practice. New York: Haffner, p. 123-130.

Passet, R. (2000). L'illusion néoliberale. Paris : Fayard. Passet, R. (1979). L'économique et le vivant. Paris : Payot.

Pineau, G., (dir.). (2001a). Pour une écoformation. Former à et par l'environnement. Éducation Permanente, 148.

Pineau, G. (2001b). Introduction générale. In R. Barbier et G. Pineau (dir.), Les eaux écoformatrices. Paris : L'Harmattan, p. 15-22.

Pineau, G. (1992). De l'air. Essai sur l'écoformation. Paris : Païdeia.

Prades, J.A. (1995). L'éthique de l'environnement et du développement. Paris : PUF.

Prigogine, I. (1996). La fin des certitudes. Temps, chaos et les lois de la nature. Paris : Odile Jacob. Ragou, P. et Souchon, C. (2000). L'approche systémique : une méthode d'apprentissage et un outil d'évaluation pédagogique en éducation relative à l'environnement. Éducation relative à l'environnement : Regards - Recherches - Réflexions, 2, 195-200.

Reigota, M. (1995). Meio ambiente e representação social. São Paulo : Cortez.

Ribeiro, D. (1981). O processo civilizatório. Etapas da evolução sociocultural. Estudos de antropologia da civilização. Petrópolis : Vozes.

Robottom, I. et Hart, P. (1993). Research in environmental education. Engaging the debate. Victoria : Deakin University.

Sachs, I. (1995). À la recherche de nouvelles stratégies de développement. Enjeux du Sommet Social. Paris : UNESCO.

Sachs, I. (dir.). (1981). Initiation à l'écodéveloppement. Toulouse : Privat.

Sachs, I. (1980). Stratégies de l'écodéveloppement. Paris : Les Éditions Ouvrières.

Sader, E. (2000). Século XX. Uma biografia não-autorizada. O século do imperialismo. São Paulo : Editora Fundação Perseu Abramo.

Sauvé, L. (2001). Recherche et formation en éducation relative à l'environnement : une dynamique réflexive. Éducation Permanente, 148, 31-44.

Sauvé, L. (1999). Un « patrimoine » de recherche en construction. Éducation relative à l'environnement : Regards - Recherches - Réflexions, 1, 13-40.

Sauvé, L. (1997). Pour une éducation relative à l'environnement. Montréal : Guérin.

Sauvé, L. (1996). Éducation relative à l'environnement : pour un savoir critique et un agir responsable. In R. Tessier et J.-G. Vaillancourt (dir.), La recherche sociale en environnement. Nouveaux paradigmes. Montréal : Les Presses de l'Université de Montréal, p. 89-106. 
Schweitzer, A. (1976). Die Lehre von der Ehrfurcht vor dem Leben und ihre Bedeutung für unsere Kultur. In H.W. Bähr (dir.), Die Lehre von der Ehrfurcht vor dem Leben. Grundtexte aus fünf Jahrzehnten. München : Beck Verlag, p. 121-143.

Singh, S., Sankaran, V., Mander, H. et Worah, S. (2000). Strenghtening conservation cultures. Local communities and biodiversity conservation. Paris : UNESCO-MAB.

Sjöberg, L. (1988). Psychological perspectives on environmental problems. In U. Svedin et B. Heurling (dir.), Human response to global change. Stockholm : SCPCR, p. 69-99.

Trajber, R. et Costa, L.B. (dir.). (2001). Avaliando a educação ambiental no Brasil. Materiais audiovisuais. São Paulo : Peirópolis.

UNESCO. (1996). From a culture of violence to a culture of peace. Paris : UNESCO.

Varela, F.J. (1996). Quel savoir pour l'éthique? Action, sagesse et cognition. Paris : La Découverte. Varela, F.J. (1989). Autonomie et connaissance. Essai sur le vivant. Paris : Seuil.

Varela, F.J., Thompson, E. et Rosch, E. (1993). L'inscription corporelle de l'esprit. Sciences cognitives et expérience humaine. Paris : Seuil.

Vieira, P.F. (dir.). (2003). Conservação da diversidade biológica e cultural em zonas costeiras. Enfoques e experiências na América Latina e no Caribe. Florianópolis : APED Editora.

Vieira, P.F. (1998). Social sciences and environment in Brazil. A state of the art report. Paris : UNESCO.

Vieira, P.F. et Weber, J. (dir.). (2002). Gestão de recursos naturais renováveis e desenvolvimento. Novos desafios para a pesquisa ambiental. São Paulo : Cortez. Vivien, F.D. (1994). Économie et écologie. Paris : La Découverte.

Von Bertalanffy, L. (1968). General systems theory. New York : Braziller.

Weber, J. (2002). Gestão de recursos renováveis : fundamentos teóricos de um programa de pesquisas. In P.F. Vieira et J. Weber (dir.), Gestão de recursos naturais renováveis e desenvolvimento. Novos desafios para a pesquisa ambiental. São Paulo : Cortez, p. 115-146.

Weil, P. (2001). Educação para a paz e o meio ambiente. Marco Social, 3, 18-23. Weil, P. (1987). Nova linguagem holística. Rio de Janeiro : Espaço e Tempo.

Woodcock, G. (1975). As idéias de Gandhi. São Paulo : Cultrix.

\section{RÉSUMÉS}

Cet article rend compte d'une réflexion exploratoire sur les fondements conceptuels, la pertinence et les préalables de la démarche d'éducation pour l'écodéveloppement. Un bilan critique des activités d'éducation relative à l'environnement au Brésil pendant la dernière décennie nourrit l'argumentation. L'auteur associe la pédagogie de l'écodéveloppement à la prise en compte de l'interdépendance économique, politique, culturelle et écologique du monde moderne, de façon à stimuler la quête de lucidité au regard de l'unité du vivant et le sens de la compassion et de la non-violence active. Il témoigne aussi de l'importance du développement d'une nouvelle conception de la formation de formateurs en ce domaine.

This paper is based on an exploratory reflection on the conceptual bases, the relevance and the prerequisites of ecodevelopment education. The argumentation is inspired by a critical assessment of environmental education activities undertaken in Brazil during the last decade. The author associates the pedagogy of ecodevelopment with the consideration of the economic, 
political, cultural and ecological interdependence of the modern world, in order to stimulate the search for lucidity while taking under account the respect for life, compassion and active nonviolence. The author also highlights the importance of developing a new conception of teacher training in this field.

\section{INDEX}

Keywords : Ecodevelopment, systemic approach, environmental sciences, ecology, environmental education, heritage, research-action-training

Mots-clés : Écodéveloppement, approche systémique, sciences de l'environnement, écologie, éducation relative à l'environnement, patrimoine, recherche-action-formation

Index géographique : Brésil

\section{AUTEUR}

\section{PAULO FREIRE VIEIRA}

Professeur au programme de doctorat en sociologie politique à l'Université Fédérale de Santa Catarina, Brésil et formé en philosophie et pédagogie, il a obtenu son doctorat en sociologie politique à l'Université de Munich (Allemagne) et a réalisé plusieurs stages postdoctoraux en écologie humaine à l'École des Hautes Études en Sciences Sociales (France). Il s'intéresse aussi à l'épistémologie des sciences de l'environnement et concentre actuellement ses activités de recherche dans le domaine de l'éducation pour l'écodéveloppement. 